\title{
Amamentação e o desenvolvimento pondo-estatural do lactente até o sexto mês de vida
}

\section{Breastfeeding and the weight-height development of the infant until the sixth month of life}

\author{
Cláudia Silveira Viera ${ }^{1}$, Luana Cecília Rocha ${ }^{2}$, Marialda Christoffel ${ }^{3}$, \\ Beatriz Rosana Gonçalves de Oliveira Toso ${ }^{4}$, Janaine Fragnan Peres ${ }^{5}$
}

\section{Resumo}

Objetivo: comparar o crescimento pondo-estatural dos lactentes aos seis meses de vida em aleitamento materno exclusivo e aleitamento complementar ou misto. Método: estudo transversal, com 38 mães e lactentes entre o quinto e o sexto mês de vida, cuja coleta de dados ocorreu em unidades de saúde de um município no Oeste do estado do Paraná, Brasil. Os dados foram analisados por estatística descritiva. Resultados: a prevalência do aleitamento materno exclusivo foi de $21 \%(n=8)$ da amostra. As variáveis antropométricas (peso, altura e perímetro cefálico - PC) dos lactentes em aleitamento materno exclusivo apresentaram médias inferiores àqueles em aleitamento materno predominante e/ou com uso de fórmula. Conclusão: o crescimento pondo-estatural dos lactentes aos seis meses de vida em aleitamento materno exclusivo e em aleitamento complementar ou misto estava dentro do esperado para a idade. Contudo, evidenciou-se baixa prevalência de aleitamento materno exclusivo.

Palavras-chave: Aleitamento materno; Alimentação artificial; Desmame precoce; Crescimento; Lactente.

\begin{abstract}
Objective: compare the weight-height growth of the infants at six months of life in exclusive breastfeeding and complementary or mixed breastfeeding. Method: transversal study, with 38 mothers and infants between the fifth and the sixty months of life, whose data collect occurred in primary health care units of a city in the West of the state of Paraná, Brazil. The data was analyzed by descriptive statistics. Results: the prevalence of exclusive breastfeeding was of $21 \%(n=8)$ of the sample. The anthropometrics variables (weight, height and head circumference - HC) of the infants in exclusive breastfeeding show lower averages to those in predominant breastfeeding and/or with use of formula. Conclusion: the weight-height growth of the infants at six months of life in exclusive breastfeeding and in complementary or mixed breastfeeding was inside of the expected for age. However, was evidenced low prevalence of exclusive breastfeeding.
\end{abstract}

Keywords: Breastfeeding; Artificial feeding; Early weaning; Growth; Infant.

\footnotetext{
${ }^{1}$ Doutorado em Enfermagem em Saúde Pública pela Escola de Enfermagem de Ribeirão Preto da Universidade de São Paulo (EERP-USP), Ribeirão Preto, São Paulo, Brasil. Professora do Curso de Enfermagem (Área de Saúde da Criança e do Adolescente) na Universidade Estadual do Oeste do Paraná (Unioeste), Cascavel, Paraná, Brasil.E-mail: clausviera@gmail.com

${ }^{2}$ Graduanda em Enfermagem na Universidade Estadual do Oeste do Paraná, Cascavel, Paraná, Brasil.

${ }^{3}$ Doutorado em Saúde de Grupos Humanos, Saúde da Criança e Adolescentes pela Escola de Enfermagem Anna Nery (EEAN), Rio de Janeiro, Rio de Janeiro, Brasil. Professora do Curso de Enfermagem na Escola de Enfermagem Anna Nery, Rio de Janeiro, Rio de Janeiro, Brasil.

${ }^{4}$ Doutorado em Enfermagem em Saúde Pública pela Escola de Enfermagem de Ribeirão Preto da Universidade de São Paulo, Ribeirão Preto, São Paulo, Brasil. Professora do Curso de Enfermagem (Área de Saúde da Criança e do Adolescente) na Universidade Estadual do Oeste do Paraná, Cascavel, Paraná, Brasil.

${ }^{5}$ Mestrado em Biociências e Saúde pela Universidade Estadual do Oeste do Paraná, Cascavel, Paraná, Brasil. Enfermeira do Consórcio Intermunicipal de Saúde do Oeste do Paraná (Cisop), Cascavel, Paraná, Brasil.
} 


\section{Introdução}

O aleitamento materno exclusivo (AME) é o evento mais impactante durante os primeiros meses de vida dos lactentes. Essa prática deve ser realizada de modo exclusivo, conforme o recomendado pela Organização Mundial da Saúde (OMS), até os seis meses de idade. Somente após esse período deve ser iniciada a introdução da alimentação complementar, com alimentos saudáveis, mantendo o aleitamento materno até os dois anos de vida. O AME, além de servir como fator protetor de enfermidades tanto na infância quanto na vida adulta, se torna um potencializador do processo de crescimento e desenvolvimento infantil. ${ }^{(1)}$

A amamentação é caracterizada como uma prática eficaz para a promoção de vínculo entre o binômio mãe-filho, propiciando afeto, proteção e nutrição para o lactente, prevenindo potencialmente o sobrepeso, a obesidade e a desnutrição e influenciando diretamente na redução da morbimortalidade infantil. ${ }^{(2)}$

Um estudo ocorrido na Galícia demonstrou que a prevalência do AME foi de $52 \%$ na $16^{\mathrm{a}}$ semana após o parto, enquanto no sexto mês era de somente $28,2 \%$. Nessa mesma pesquisa, observou-se que as mulheres que pretendiam alimentar os bebês com outro tipo de aleitamento possuíam menor conhecimento quanto ao aleitamento materno. ${ }^{(3)}$

Outra pesquisa realizada com objetivo de observar a prevalência dos indicadores de aleitamento materno e AME no Brasil durante o período de 1986 a 2013, mostrou que ambos apresentaram índices de crescimento. A amamentação exclusiva ampliou-se de $2,9 \%$ para $37,1 \%$ de 1986 a 2006, mantendo essa média entre 2006 e 2013. ${ }^{(4)} \mathrm{O}$ relatório parcial do Estudo Nacional de Alimentação e Nutrição Infantil (ENANI) demonstrou que houve aumento da prevalência de aleitamento materno entre as crianças brasileiras avaliadas entre 2019 e 2020 evidenciando que 45,7\% destas estavam em AME ate o sexto mês de vida. ${ }^{(5)}$

Apesar de ter sido evidenciado esse aumento do AME no relatório ENANI, a prevalência continua inferior a $50 \%$; portanto, mais da metade das crianças não são amamentadas com leite materno nesse período. O desmame precoce incorre em maior risco para déficit de crescimento, visto que os escores Z médios mais altos de Índice de Massa Corpórea (IMC) são observados naquelas crianças que não são amamentadas exclusivamente com leite materno. ${ }^{(6)}$ Constata-se nessa relação que a maior duração do AME é fator de proteção contra a obesidade infantil. ${ }^{(7)}$

Outro aspecto relevante é a relação do AME e a velocidade de crescimento infantil, em que essa velocidade é maior entre as crianças em aleitamento exclusivo ou predominante quando comparadas àquelas com consumo de fórmula láctea infantil. ${ }^{(7)}$ Outro achado importante foi que os filhos das mães que relataram dificuldade com a amamentação tinham menor velocidade de crescimento. ${ }^{(8)}$ Porém, outro estudo apontou que não foi observada correlação do crescimento com a duração do AME após os seis meses de vida do lactente. ${ }^{(9)}$

A gestante e a família devem ser orientadas sobre os benefícios da amamentação desde o início do pré-natal, dentre eles acerca do crescimento saudável e redução do risco de obesidade futura. Essa ação deverá ser realizada por profissionais de saúde capacitados, de modo que a mulher, sua família e a sociedade percebam como o leite materno é essencial e o período em que a prática da amamentação é crucial para o crescimento da criança. ${ }^{(1)}$

Com base no exposto, este estudo tem por objetivo comparar o crescimento pondo-estatural dos bebês aos seis meses de vida que estiverem em aleitamento materno exclusivo com aqueles que estiverem em aleitamento complementar ou misto.

\section{Método}

Este estudo é integrante de um projeto multicêntrico internacional intitulado "Lactância materna exclusiva: determinantes socioculturales en Latino América", sob coordenação da University of Kentucky (UK), Estados Unidos, sendo que no contexto do Brasil o subprojeto foi denominado "Aleitamento materno exclusivo: determinantes socioculturais no Brasil". 
Trata-se de estudo de abordagem quantitativa, de desenho transversal, em que se buscou a relação entre o tipo de aleitamento recebido por lactentes até o sexto mês de vida (variável que influenciará para o desfecho) e o crescimento pondoestatural (fenômeno/desfecho estudado).

Os dados foram coletados em unidades de saúde de município do Sul do Brasil, no período de janeiro a março de 2020 junto a lactentes que tivessem entre cinco e seis meses de vida, durante consultas médicas, de puericultura ou vacinação, acompanhados de suas mães e/ou cuidadores. A amostra final foi de 38 lactentes, tendo-se como limitante para o " $\mathrm{n}$ " amostral o fechamento das unidades de saúde devido à pandemia da Covid-19.

$\mathrm{O}$ instrumento pré-elaborado para o projeto multicêntrico foi ajustado para a coleta de dados desta pesquisa envolvendo as variáveis: tipo de aleitamento entre cinco e seis meses (exclusivamente leite materno; predominantemente leite materno; alimentação mista e predominantemente leite artificial) e antropométricas do lactente (peso, estatura e perímetro cefálico - PC), a partir do registro da carteira da criança e da entrevista com o cuidador.
Os dados foram tabulados no programa Excel $^{\circledR}$ for Windows ${ }^{\circledR}$ por conferência dupla para análise estatística descritiva, com frequência absoluta, frequência relativa, média e desvio-padrão (DP).

A pesquisa foi aprovada pelo Comitê de Ética em Pesquisa com Seres Humanos, sob parecer $n^{\circ}$ 2.507.525 e CAAE $n^{\circ}$ 80711517.8.1001.5238, e, ao ser informado sobre o estudo, cada participante recebeu o Termo de Consentimento Livre e Esclarecido (TCLE) em duas vias, para assinar, ficando com uma delas e a outra com o pesquisador.

\section{Resultados}

Do total de crianças avaliadas no seguimento entre o quinto e sexto mês ( $\mathrm{n}=38$ ), a média de dias de vida na entrevista foi de 170,76 ( $\mathrm{DP}=13,47)$, o peso médio foi 7.335,19 g $(\mathrm{DP}=899,69)$, a média da estatura foi de $65,52 \mathrm{~cm}(\mathrm{DP}=2,86)$ e o PC teve uma média de 43,26 cm (DP=1,40).

Na Tabela 1 apresenta-se a caracterização das crianças avaliadas em relação ao tipo de alimento recebido no momento da entrevista.

Tabela 1 - Caracterização das crianças avaliadas quanto ao tipo de alimentação recebida no momento da entrevista. Cascavel, Paraná, Brasil, 2020.

\begin{tabular}{lcc}
\hline Tipo de alimentação & Frequência absoluta (N) & Frequência relativa (\%) \\
\hline Exclusivamente leite materno & 8 & 21,0 \\
Predominantemente leite materno & 19 & 50,0 \\
Alimentação mista & 2 & 5,3 \\
Predominantemente leite artificial & 9 & 23,7 \\
\hline Total & 38 & 100,0 \\
\hline
\end{tabular}

Fonte: dados da pesquisa.

O AME estava presente para apenas $8(21 \%)$ das crianças avaliadas no período; a maioria das entrevistadas estava em aleitamento materno predominante $(50 \%)$.

Dentre os lactentes analisados $(\mathrm{n}=38)$, foi obtido o peso de $27(71,0 \%)$ destes, e o mesmo variou entre $5.800 \mathrm{~g}$ e $9.020 \mathrm{~g}$. A estatura de $25(65,8 \%)$ crianças foi registrada, estando entre $60 \mathrm{~cm}$ e 73 $\mathrm{cm}$. O perímetro cefálico também foi observado em $65,8 \%$ dos entrevistados, com variação entre $41 \mathrm{~cm}$ e $46 \mathrm{~cm}$. Não foram obtidas as variáveis antropométricas daqueles em alimentação mista.

Como apresentado anteriormente, as médias das medidas antropométricas, independentemente 
do tipo de aleitamento recebido pelas crianças em estudo, estão incluídas entre o escore $\mathrm{Z}$ que se espera na faixa de cinco a seis meses de idade. ${ }^{(8-9)}$
Nas Figuras 1, 2 e 3 pode ser observado o tipo de aleitamento recebido com relação às variáveis peso, estatura e perímetro cefálico.

Figura 1 - Tipo de aleitamento materno e a variável peso (N=27). Cascavel, Paraná, Brasil, 2020.

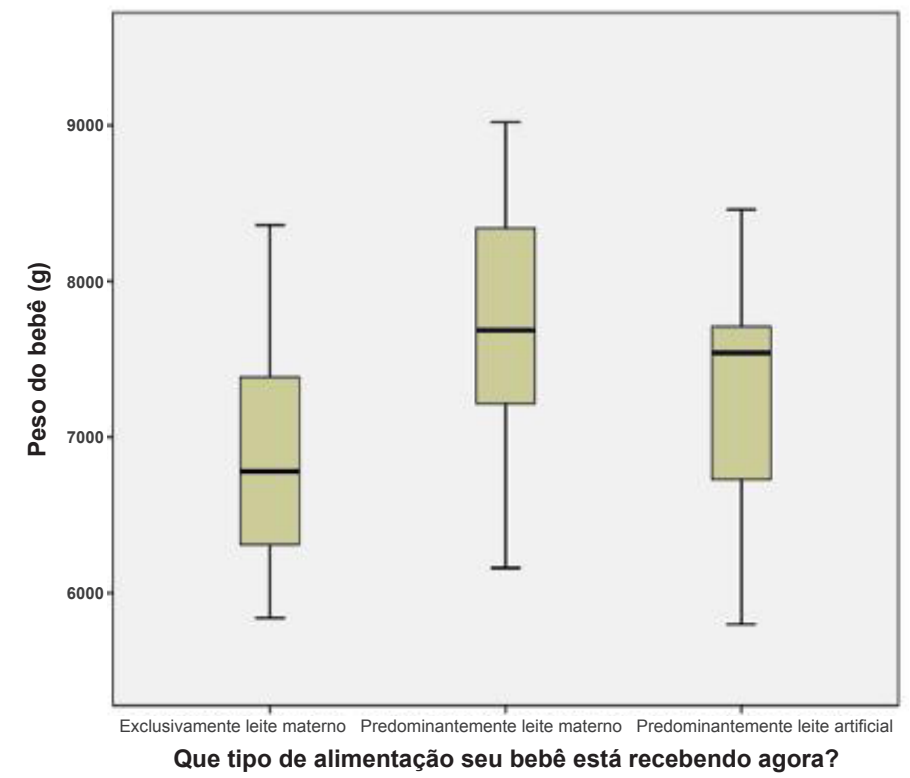

Fonte: dados da pesquisa.

Como se verifica na Figura 1, o peso para os lactentes em AME apresentou média de $6.910 \mathrm{~g}$ $(\mathrm{DP}=861,57)$, sendo que o peso mínimo foi 5.840 g e o máximo 8.360 g. Para os bebês com aleitamento materno predominante obteve-se média de
$7.673,33 \mathrm{~g}(\mathrm{DP}=835,84)$; o valor mínimo foi 6.160 g e o máximo 9.020 g. Por sua vez, a variação de peso para aqueles em alimentação por leite artificial predominantemente ocorreu entre $5.800 \mathrm{~g}$ e $8.460 \mathrm{~g}$; a média foi $7.296,67 \mathrm{~g}(\mathrm{DP}=921,19)$.

Figura 2 - Tipo de aleitamento materno e a variável estatura (N=25). Cascavel, Paraná, Brasil, 2020.

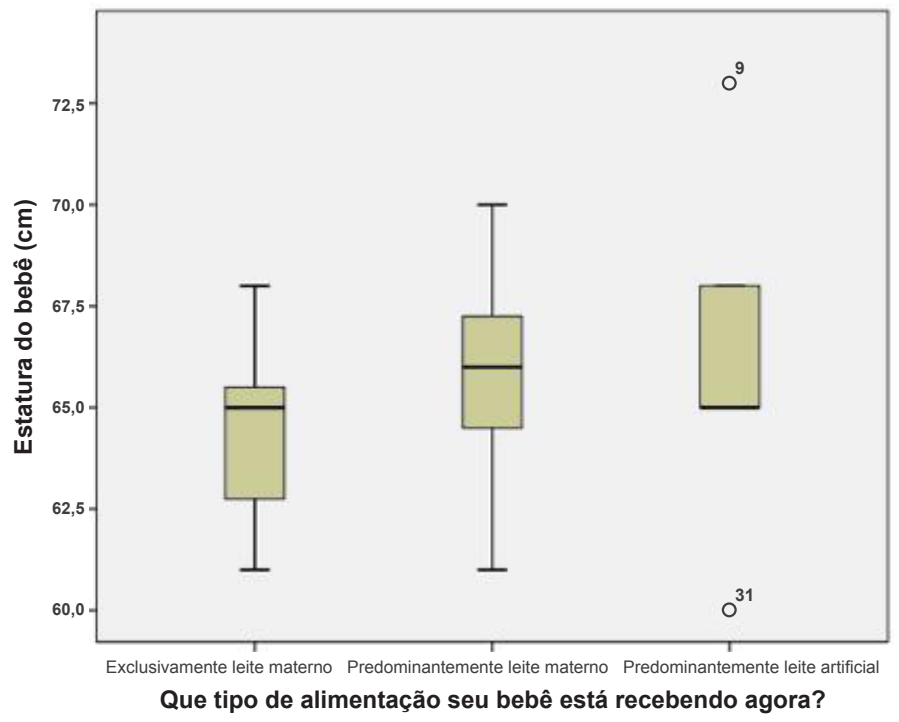

Fonte: dados da pesquisa. 
A variável estatura, como demonstrado na Figura 2, apresentou média de 64,36 cm $(\mathrm{DP}=2,36)$ para as crianças que ainda estavam em AME, com variação entre $61 \mathrm{~cm}$ e $68 \mathrm{~cm}$. Os lactentes em aleitamento materno predominante obtiveram média de estatura de $65,96 \mathrm{~cm}(\mathrm{DP}=2,30)$, sendo o valor mínimo de $61 \mathrm{~cm}$ e o máximo de $70 \mathrm{~cm}$. Já os bebês que se encontravam em alimentação predominante de leite artificial tiveram média de 66 $\mathrm{cm}(\mathrm{DP}=4,29)$, com variação entre $60 \mathrm{~cm}$ e $73 \mathrm{~cm}$.

Figura 3 - Tipo de aleitamento materno e a variável perímetro cefálico $(\mathrm{N}=25)$. Cascavel, Paraná, Brasil, 2020.

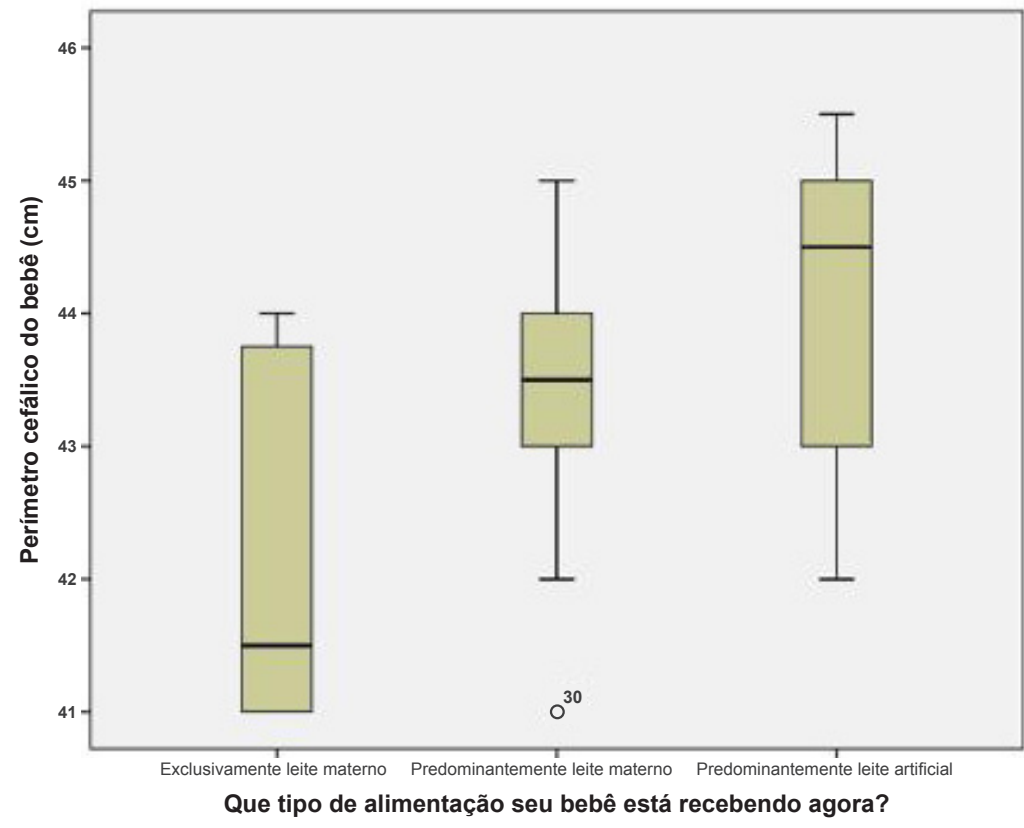

Fonte: dados da pesquisa.

Como é possível observar na Figura 3, os bebês que ainda estavam em alimentação exclusiva de leite materno obtiveram média de perímetro cefálico igual a $42,29 \mathrm{~cm}(\mathrm{DP}=1,47)$, tendo variação de $41 \mathrm{~cm}$ a $44 \mathrm{~cm}$. Já aqueles com leite materno predominante como alimentação obtiveram média de 43,42 cm $(\mathrm{DP}=1,14)$ e os dados variaram entre $41 \mathrm{~cm}$ e $45 \mathrm{~cm}$. Entre as crianças que estavam recebendo leite artificial predominantemente houve variação de $42 \mathrm{~cm}$ a $46 \mathrm{~cm}$ no perímetro cefálico, sendo de 44,08 $\mathrm{cm}$ a média ( $\mathrm{DP}=1,36)$.

Com base nas Figuras 1, 2 e 3 percebe-se, portanto, que as variáveis antropométricas peso, estatura e perímetro cefálico não apresentaram médias maiores para as crianças que estavam em AME. Apesar disso, observou-se que os valores se encontravam dentro da faixa de escore $\mathrm{Z}$ esperado para a idade entre cinco e seis meses de vida. ${ }^{(8-9)}$

\section{Discussão}

A análise dos dados evidenciou que há uma baixa prevalência de AME (21\%) ao sexto mês entre os lactentes do estudo, sendo o período inferior ao recomendado pelo Ministério da Saúde (MS) e pela OMS. Observa-se, portanto, que existe um alto índice de desmame precoce entre as mulheres entrevistadas. Dado semelhante foi encontrado em estudo realizado no município de Sorocaba, estado de São Paulo, Brasil, no qual 22\% das mulheres avaliadas amamentaram de modo exclusivo nos seis primeiros meses de vida. ${ }^{(10)}$ Ambos dados inferiores à prevalência de AME em todas as regiões brasileiras em 2015, identificada como de 54\%. ${ }^{(11)}$

$\mathrm{O}$ desmame precoce pode estar associado a diversos fatores sociais, culturais, econômicos e de assistência à saúde. Isso demonstra que para 
a redução do índice de desmame precoce e, como consequência, aumento da prevalência de AME, é preciso estimular ainda mais a promoção do AME, desde o pré-natal, nas unidades de Atenção Primária à Saúde (APS), mas que haja continuidade após o parto na hospitalização e durante o puerpério, nas consultas e visitas domiciliares. ${ }^{(12)}$

Outro aspecto observado no atual estudo foi que os dados antropométricos dos lactentes amamentados exclusivamente até os seis meses de vida apresentaram valores inferiores aos demais tipos de alimentação.

Essa comparação entre o peso dos lactentes em AME e alimentados com leite artificial está em consonância com um estudo de revisão não sistemática que observou que lactentes em AME ganham menos peso entre os três e seis meses de idade em relação aos lactentes alimentados com fórmula. ${ }^{(13)}$ Enfatiza-se que o AME é um protetor para obesidade e diabete mellitus, seja na infância, adolescência ou mesmo idade adulta. ${ }^{(14)}$ Portanto, embora o crescimento de lactentes possa ser inferior aos demais tipos de aleitamento, as crianças em AME estarão mais protegidas em sua saúde, em específico para reduzir o risco de obesidade tardia, ${ }^{(7)}$ além de reduzir o risco de hospitalizações por infecções de vias aéreas superiores e acometimentos por problemas gastrointestinais, como a diarreia, entre outros problemas. ${ }^{(15)}$

$\mathrm{O}$ presente estudo mostrou ainda que as variáveis antropométricas analisadas nos lactentes amamentados até o sexto mês exclusivamente estavam dentro da faixa de escore $\mathrm{Z}$ esperado para a idade, o que indica um crescimento saudável e de acordo com os valores de referência da OMS. ${ }^{(16)}$

A partir de uma rede intensiva de aconselhamento familiar foram avaliados a amamentação e o crescimento infantil em regiões de Bangladesh, ${ }^{(17)}$ evidenciando-se que dos bebês com peso normal ao nascimento amamentados exclusivamente com leite materno pelo período recomendado, aos seis meses tinham dados de peso semelhantes aos valores de referência da OMS. Ainda, observaram que os lactentes com baixo peso ao nascer, também amamentados exclusivamente até os seis meses de vida, tiveram redução drástica da prevalência de baixo peso, visto que no primeiro mês era de $42,1 \%$ e ao sexto mês era de apenas $21,1 \%$. $^{(17)}$

Percebe-se que o AME durante o tempo preconizado e sua continuidade com complementação até os dois anos ou mais, é essencial para o crescimento saudável dos bebês, refletindo em sua vida em curto e longo prazo, uma vez que reduz o risco ao adoecimento infantil. Em avaliação de crianças de dois a quatro anos em centros de educação infantil (CEIs) do município de Taubaté, estado de São Paulo, Brasil, observou-se que, quanto mais precoce a introdução de outro leite na dieta dessas crianças, maior era a correlação com o excesso de peso na idade pré-escolar, relacionando-se essa introdução com a diminuição do efeito protetor do aleitamento materno quanto ao risco de desenvolvimento de sobrepeso e obesidade. ${ }^{(18)}$

Informações similares foram obtidas em um ambulatório de uma maternidade filantrópica e em uma clínica privada no estado de Sergipe, Brasil, em que, relacionando os tipos de alimentação dos lactentes nos seis primeiros meses de idade com seu estado nutricional, evidenciou-se melhor estado nutricional entre aqueles amamentados exclusivamente até os seis meses comparado àqueles que já possuíam introdução de outros alimentos antes desse período. Demonstrou-se que a maioria das crianças em AME possuía IMC adequado para a idade, além de menor classificação para baixo peso, e nenhuma classificada com obesidade, indicando a importância da amamentação exclusiva na prevenção da desnutrição e da obesidade durante a infância. ${ }^{(19)}$

Apresentou-se baixa prevalência de AME entre cinco e seis meses, o que indica que houve desmame precoce entre crianças avaliadas. Desse modo, é preciso que os profissionais de saúde, e principalmente os enfermeiros, compreendam a importância do AME para um sadio crescimento e desenvolvimento dos lactentes durante os seus primeiros meses de vida e promovam estratégias que estimulem a manutenção do AME por maior 
tempo. Assim, sugere-se que estudos devam ser realizados com intuito de identificar as causas de desmame precoce entre as nutrizes do município para que se possa planejar intervenções de promoção do AME e, dessa forma, se promova o crescimento saudável de lactentes pelas boas práticas alimentares, contribuindo para redução da obesidade e condições crônicas de saúde na vida adulta.

\section{Conclusão}

Os resultados do estudo evidenciaram que independentemente do tipo de aleitamento recebido pelas crianças entre cinco e seis meses de vida, houve crescimento adequado para idade de todas as crianças participantes do estudo. Constatou-se que não houve maior ganho pondo-estatural e do perímetro cefálico entre os que estavam em AME, o que foi observado para aqueles que recebiam predominantemente leite materno, visto que tiveram médias maiores quando comparados com os outros tipos de aleitamento.

\section{Agradecimentos}

Ao Conselho Nacional de Desenvolvimento Científico e Tecnológico (CNPq) pelo apoio financeiro recebido.

\section{Referências}

1 Ministério da Saúde - MS (BR). Agenda de compromissos para a saúde integral da criança e redução da mortalidade infantil [Internet]. Brasília: Ministério da Saúde; 2004 [citado 2020 jul 30]. Disponível em: http://bvsms.saude.gov. br/bvs/publicacoes/agenda_compro_crianca.pdf

2 Ministério da Saúde - MS (BR). Saúde da criança: aleitamento materno e aleitamento complementar. 2a ed. [Internet]. Brasília: Ministério da Saúde; 2015 [citado 2020 jul 30]. Disponível em: https://bvsms.saude.gov. $\mathrm{br} / \mathrm{bvs} /$ publicacoes/saude_crianca_aleitamento_ materno_cab23.pdf
3 Suárez-Cotelo MC, Movilla-Fernández MJ, Pita-García P, Arias BF, Novío S. Breastfeeding knowledge and relation to prevalence. Rev. Esc. Enferm. USP [Internet]. 2019 [citado 2020 jul 30]; (53). Disponível em: doi: 10.1590/ S1980-220X2018004503433.

4 Boccolini CS, Boccolini PMM, Monteiro FR, Venâncio SI, Giugliani ERJ. Breastfeeding indicators trends in Brazil for three decades. Rev. SaudePublica [Internet]. 2017 [citado 2020 jul 30]; 51(108). Disponível em: doi: 10.11606/ S1518-8787.2017051000029.

5 Universidade Federal do Rio de Janeiro. Estudo Nacional de Alimentação e Nutrição Infantil - ENANI-2019: Resultados preliminares Indicadores de aleitamento materno no Brasil. Rio de Janeiro: UFRJ, 2020.

6 Weber M, Grote V, Closa-Monasterolo R, Escribano J, Langhendries J, Dain E, et al. Lower protein content in infant formula reduces BMI and obesity risk at school age: follow-up of a randomized trial. Am. J. Clin. Nutr. [Internet]. 2014 [citado 2020 ago 4]; (5). doi: 10.3945/ajen.113.064071.

7 Carling SJ, Demment MM, Kjolhede CL, Oslon CM. Breastfeeding Duration and Weight Gain Trajectory in Infancy. Pediatrics [Internet]. 2015 [citado 2020 ago 4]; 135(1). doi: 10.1542/ peds.2014-1392.

8 Fonseca PCA, Carvalho CA, Ribeiro SAV, Nobre LN, Pessoa MC, Ribeiro AQ, et al. Determinantes da velocidade média de crescimento de crianças até seis meses de vida: um estudo de corte. Ciênc. Saúde Coletiva [Internet]. 2017 Ago [citado 2020 set 2]; 22(8): 2713-26. Disponível em: http:// www.scielo.br/scielo.php? script $=$ sci arttext\&pid=S $1413-8123201700280$ $2713 \& \operatorname{lng}=\mathrm{pt}$

9 Ferreira PVA, Leal VS, Silva MMC, Mukai AO, Rodrigues CLS, Bertoli CJ, et al. Crescimento de lactentes durante o primeiro ano de vida. J. Hum. Growth Dev. [Internet]. 2015 [citado 2 set 2020]; 25(2):211-215. Disponível em: http://www.revistas.usp.br/jhgd/article/ view/103017/103056 
10 Silva AC, Barros DP. Assistência de enfermagem em puericultura: fatores que influenciam o desmame precoce. Enferm. Bras. [Internet]. 2019 [citado 2020 jul 30]; 18(2). doi: 10.33233/eb.v18i2.2512.

11 Nascimento JC, Silva NL, Lima MFS, Lima MCBM, Oliveira GS. Prevalência do aleitamento materno exclusivo nas regiões brasileiras em 2015. Carpe Diem [Internet]. 2018 [citado 2020 ago 4]; 16(2). Disponível em: https://periodicos.unifacex.com.br/Revista/ article/view/1020.

12 Santos PV, Martins MCC, Tapety FI, Paiva AA, Fonseca FMNS, Brito AKS. Desmame precoce em crianças atendidas na Estratégia Saúde da Família. Rev. Eletr. Enf. [Internet]. 2018 [citado 2020 jul 30]; (20). doi: 10.5216/ ree.v20.43690.

13 Giugliani ERJ. Growth in exclusively breastfed infants. J. Pediatr. [Internet]. 2019 [citado 2020 jul 30]; 95(Suppl 1). doi: 10.1016/j. jped.2018.11.007.

14 Horta BL, Mola CL, Victora CG. Long-term consequences of breastfeeding on cholesterol, obesity, systolic blood pressure and type 2 diabetes: a systematic review and metaanalysis. Acta Paediatr. [Internet]. 2015 [citado 2020 jul 30]; (104). doi: 10.1111/apa.13133.

15 Frank NM, Lynch KF, Uusitalo U, Yang J, Lönnrot M, Virtanen SM, et al. The relationship between breastfeeding and reported respiratory and gastrointestinal infection rates in young children. BMC Pediatr. [Internet]. 2019 [citado 2020 jul 30]; 19(339). doi: 10.1186/s12887019-1693-2.

16 World Health Organization (WHO). WHO Child Growth Standards. Length/height-forage, weight-for-age, weight-for-length, weightfor-height and body mass index-for-age: Methods and development. [Internet] Geneva: WHO; 2006 [citado 2020 ago 4]. Disponível em: https://www.who.int/childgrowth/ standards/Technical_report.pdf

17 Haider R, Saha KK. Breastfeeding and infant growth outcomes in the context of intensive peer counselling support in two communities in Bangladesh. Int. Breastfeed. J. [Internet]. 2016 [citado 2020 jul 30]; 11(18). doi: 10.1186/ s13006-016-0077-6.

18 Nascimento VG, Silva JPC, Ferreira PC, Bertoli CJ, Leone C. Maternal breastfeeding, early introduction of non-breast milk, and excess weight in preschoolers. Rev. Paul. Pediatr. [Internet]. 2016 [citado 2020 jul 30]; 34(4). doi: 10.1016/j.rppede.2016.05.002.

19 Santos AJAO, Bispo AJB, Cruz LD. Padrão de aleitamento e estado nutricional de crianças até os seis meses de idade. H.U. Revista [Internet]. 2016 [citado 2020 jul 30]; 42(2). Disponível em: https://periodicos.ufjf.br/index.php/ hurevista/article/view/2514 